\title{
AN ALMOST SURE INVARIANCE PRINCIPLE FOR HILBERT SPACE VALUED MARTINGALES
}

BY

\author{
GREGORY MORROW AND WALTER PHILIPP
}

\begin{abstract}
We obtain an almost sure approximation of a martingale with values in a real separable Hilbert space $H$ by a suitable $H$-valued Brownian motion.
\end{abstract}

1. Introduction. In his fundamental papers Strassen $[15,16]$ established almost sure invariance principles first for sums of independent identically distributed random variables with zero means and unit variances and later for certain classes of martingales. These are almost sure approximations of such sums or martingales by standard Brownian motion. Several authors subsequently built upon this foundation by producing invariance principles for sums of weakly dependent random variables. A crucial step in common to the proofs of these results is an approximation of the partial sums by a martingale to which the Skorohod imbedding theorem is applied. Yet as these methods rely on the Skorohod imbedding theorem their use is ostensibly restricted to real valued random variables.

To get beyond this barrier Berkes and Philipp [1] developed a different method based on the approximation of weakly dependent random vectors by independent variables having prescribed distributions. This method has been applied to obtain almost sure invariance principles for sums of mixing random variables with values in a separable Hilbert or Banach space (see [4] and [11]).

We complete here one circle in this sphere of ideas by approximating martingale differences by independent Gaussian vectors. Indeed we prove an almost sure invariance principle for martingales with values in a Hilbert space.

Let $\left\{x_{n} ; n \geqslant 1\right\}$ be a sequence of random vectors assuming values in a separable Hilbert space $(H,|\cdot|)$ with inner product $\langle\cdot, \cdot\rangle$. Let $\mathcal{L}_{n}$ be the $\sigma$-field generated by $x_{1}, \ldots, x_{n}$ and $\mathcal{L}_{0}$ the trivial $\sigma$-field. We denote by $E_{n}(\cdot)$ the conditional expectation operator $E\left(\cdot \mid \varrho_{n-1}\right)$. For the purposes of this paper we assume that $0<E\left|x_{n}\right|^{2}<\infty$ and $E_{n} x_{n}=0$ for all $n \geqslant 1$. Thus, $\left\{x_{n}, \varrho_{n} ; n \geqslant 1\right\}$ is a martingale difference sequence.

We define for each $n$ the (random) conditional covariance operator $\sigma_{n}$ :

$$
\sigma_{n}(u)=E_{n}\left(\left\langle u, x_{n}\right\rangle x_{n}\right), \quad u \in H .
$$

Received by the editors October 11, 1980.

1980 Mathematics Subject Classification. Primary 60B12; Secondary 60F17.

Key words and phrases. Invariance principles, Hilbert space, martingales, law of the iterated logarithm, central limit theorem.

'Both authors were partially supported by NSF grants. 
We have

$$
\operatorname{tr}\left(\sigma_{n}\right) \stackrel{\text { def }}{=} \sum_{i=1}^{\infty}\left\langle e_{i}, \sigma_{n}\left(e_{i}\right)\right\rangle=E_{n}\left|x_{n}\right|^{2} .
$$

Here $\left\{e_{i}\right\}$ denotes a complete orthonormal basis for $H$. Write

$$
A_{n}=\sum_{\nu \leqslant n} \sigma_{\nu}
$$

and set $V_{n}=\operatorname{tr}\left(A_{n}\right)$. We note that

$$
V_{n}=\sum_{\nu \leqslant n} E_{\nu}\left|x_{\nu}\right|^{2}
$$

We define further a seminorm $\|\cdot\|$ on linear operators $B: H \rightarrow H$ by

$$
\|B\|=\sup _{u \in H,|u|=1}|\langle u, B(u)\rangle| \text {. }
$$

Our main result is the following.

THEOREM 1. Let $\left\{x_{n}, \varrho_{n} ; n \geqslant 1\right\}$ be a martingale difference sequence with values in a real separable Hilbert space of dimension $d \leqslant \infty$. Let $f \neq \infty$ be a nondecreasing function tending to $\infty$ along the positive real axis such that $f(x) \log ^{\alpha} x / x$ is nonincreasing in $x$ for some $\alpha>50 d$ (if $d=\infty$ we interpret this last condition to mean that it holds for all large $\alpha)$. Suppose that $V_{n} \rightarrow \infty$ a.s. and

$$
D=\sum_{n \geqslant 1} E\left\{\left|x_{n}\right|^{2} 1\left(\left|x_{n}\right|^{2}>f\left(V_{n}\right)\right) / f\left(V_{n}\right)\right\}<\infty .
$$

Suppose that there is some probability measure $\mu$ on $H$ having mean zero and finite second moment so that, upon defining the operator $A$ by

$$
A(u)=\int_{H}\langle u, x\rangle x \mu(d x), \quad u \in H,
$$

we have

$$
E \sup _{n \geqslant 1} \exp \left(\left\|A_{n}-V_{n} A\right\| / f\left(V_{n}\right)\right)<\infty .
$$

Then without changing its probability law we can redefine the sequence $\left\{x_{n}, n \geqslant 1\right\}$ on a richer probability space on which there exists a mean zero Brownian motion $\{X(t) ; t \geqslant 0\}$ with values in $H$ and covariance operator $A$ such that with probability 1 ,

$$
\left|\sum_{n \geqslant 1} x_{n} \cdot 1\left(V_{n} \leqslant t\right)-X(t)\right|= \begin{cases}O\left(t^{1 / 2}(f(t) / t)^{1 / 50 d}\right) & \text { if } d<\infty \\ o\left((t \log \log t)^{1 / 2}\right) & \text { if } d=\infty\end{cases}
$$

To get some feeling for the role that $f$ plays in this theorem, observe that if $\left\{x_{n}, n \geqslant 1\right\}$ has uniformly bounded $(2+\delta)$ th moments for $0<\delta \leqslant 1$ and if $V_{n} \geqslant c n$ almost surely with some constant $c>0$ then (1.4) is satisfied with $f(x)=x^{1-\varepsilon}$ for any $0<\varepsilon \leqslant \delta / 4$. Moreover the error term in (1.6) valid for $d<\infty$ is in any case $O\left(t^{1 / 2} / \log t\right)$.

Condition (1.5) might also require some explanation. It guarantees that the averages $\left\{A_{n} / V_{n} ; n \geqslant 1\right\}$ are "close to" a nonrandom covariance operator $A$. Thus, 
condition (1.5) provides that in the limit the clock $\left\{A_{n}\right\}$, governing the martingale $\left\{\sum_{\nu \leqslant n} x_{\nu}, \mathcal{L}_{n} ; n \geqslant 1\right\}$, runs along a one-dimensional time scale.

There are several well-known applications of relation (1.6). These result from properties of Brownian motion which carry over to the process

$$
\left\{\sum_{n \geqslant 1} x_{n} \cdot 1\left(V_{n} \leqslant t\right) ; t \geqslant 0\right\}
$$

subject to the quality of the error term in (1.6). For example, without restriction on $d$ one obtains the functional law of the iterated logarithm (stated for Brownian motion in [15] when $d<\infty$ and in [10] when $d=\infty$ ) since the error term in (1.6) is always $o\left((t \log \log t)^{1 / 2}\right)$. For each $d<\infty$ one obtains an upper and lower class refinement of the classical law of the iterated logarithm since the error term $O\left(t^{1 / 2} / \log t\right)$ is sufficient for this purpose. (See for instance the proof of Theorem 5.1 in [8]. The upper and lower class refinement is formulated for $\mathbf{R}^{d}$-valued Brownian motion in Theorem 2.2 of [12]: to get a statement that is valid for $t$ near $\infty$ make a time inversion.) In the same way one obtains (with $d<\infty$ ) upper and lower class refinements of Chung's law of the iterated logarithm for the maxima of partial sums (see Theorem 6.1 of [9] for a statement of these results for Brownian motion). Also for $d<\infty$ the functional central limit theorem follows at once (divide relation (1.6) by $\sqrt{t}$ and let $t \rightarrow \infty$ ) and a central limit theorem with remainder can be derived directly from the tail estimates (2.18) below.

For $1<d \leqslant \infty$ relation (1.6) and all of the aforementioned consequences are new.

On the other hand for $d=1$ Theorem 1 is contained in Strassen's [16] Theorem 4.4. Although condition (1.5) is automatically satisfied with $A=1$, in other respects our theorem is weaker than Strassen's [16] theorem. Namely, first we assume that the series in (1.4) converges. This is in contrast to almost sure convergence of the series gotten by replacing $E(\cdot)$ with $E_{n}(\cdot)$ in the $n$th summand of (1.4). Second our class of functions $f$ is somewhat smaller and third, our error term is weaker. Yet, while in some special cases we have obtained an error term comparable to Strassen's we will not present the details since this error term is itself weaker than the one given subsequent to [16] by Jain, Jogdeo and Stout [8].

In going from $d=1$ to $d>1$ it is interesting to note that, even in the case of independent random vectors, a new phenomenon arises. As an illustration, consider a sequence $\left\{y_{n} ; n \geqslant 1\right\}$ of independent identically distributed random variables with standard normal distribution $N(0,1)$. Let $\left\{e_{1}, e_{2}\right\}$ be a set of orthogonal unit vectors in $\mathbf{R}^{2}$ and define for $2^{\nu} \leqslant n<2^{\nu+1}$ and $\nu \geqslant 0$

$$
x_{n}= \begin{cases}y_{n} e_{1} & \text { if } \nu \text { is even } \\ y_{n} e_{2} & \text { if } \nu \text { is odd }\end{cases}
$$

Then, clearly, without isolating the components of the vectors $x_{n}$ one cannot achieve a useful approximation of their partial sums by any Brownian motion in $\mathbf{R}^{2}$.

Nevertheless, for independent random vectors, even in the absence of condition (1.5), an approximation result can be obtained by introducing an $H$-valued Wiener 
process $X$ indexed by the collection $\mathcal{C}$ of nonnegative symmetric trace class operators. More precisely let $\{X(C) ; C \in \mathcal{C}\}$ be a separable Gaussian process with values in $H$ such that

(i) $X(0)=0$.

(ii) For every $C \in \mathcal{C}, X(C)$ has the Gaussian distribution with mean zero and covariance operator $C$.

(iii) $X\left(C_{1}\right), X\left(C_{1}+C_{2}\right)-X\left(C_{1}\right), \ldots, X\left(C_{1}+\cdots+C_{n}\right)-X\left(C_{1}+\cdots+C_{n-1}\right)$ are independent random vectors for any $C_{1}, \ldots, C_{n} \in \mathcal{C}$.

This process is suitable for approximating partial sums of independent random vectors even when the clock of the partial sum process runs along an infinite-dimensional time scale.

THEOREM 2. Let $\left\{x_{n} ; n \geqslant 1\right\}$ be a sequence of independent mean zero random variables with values in a real separable Hilbert space of dimension $d \leqslant \infty$. Assume the hypotheses of Theorem 1 with the possible exception of condition (1.5). Then the conclusion of Theorem 1 holds wherein an $\mathrm{H}$-valued Wiener process $X$ takes the place of Brownian motion and (1.6) is replaced by

$$
\left|\sum_{\nu \leqslant n} x_{\nu}-X\left(A_{n}\right)\right|= \begin{cases}O\left(V_{n}^{1 / 2}\left(f\left(V_{n}\right) / V_{n}\right)^{1 / 50 d}\right) & \text { if } d<\infty, \\ o\left(\left(V_{n} \log \log V_{n}\right)^{1 / 2}\right) & \text { if } d=\infty .\end{cases}
$$

For technical reasons we were unable to obtain a result for martingales corresponding to Theorem 2 with $d>1$. Condition (1.5) grew out of our efforts in this direction.

The condition $\alpha>50 d$ can be somewhat relaxed but to avoid hampering the exposition we employ the condition as stated. In $\$ 2$ we prove Theorem 1 in case $d<\infty$ and then use its proof in $\S 3$ to obtain (1.6) for $d=\infty$. The proof of Theorem 2 is similar to that of Theorem 1 so we omit it. We mention finally that the Vinogradov symbol, «, appearing below carries the same meaning as the big oh symbol of Landau.

2. Proof of Theorem $1(d<\infty)$.

2.1. Preliminaries. We begin with a few simple lemmas and definitions.

LEMMA 2.1. For any random time $\tau$ and any number $M>0$ we have

$$
P\left\{\operatorname{tr}\left(\sigma_{\tau}\right) \geqslant M \cdot f\left(V_{\tau}\right)\right\} \leqslant(1+D) M^{-1} .
$$

Proof. Evidently

$$
\begin{aligned}
\operatorname{tr}\left(\sigma_{\tau}\right) & =\operatorname{tr}\left(\sum_{n \geqslant 1} \sigma_{n} \cdot 1(\tau=n)\right) \\
& \leqslant \sum_{n \geqslant 1} \operatorname{tr}\left(\sigma_{n}\right) \cdot 1(\tau=n)=\sum_{n \geqslant 1} E_{n}\left(\left|x_{n}\right|^{2}\right) \cdot 1(\tau=n) \\
& \leqslant \sum_{n \geqslant 1} f\left(V_{n}\right)\left(1+E_{n}\left\{\left|x_{n}\right|^{2} \cdot 1\left(\left|x_{n}\right|^{2}>f\left(V_{n}\right)\right) / f\left(V_{n}\right)\right\}\right) \cdot 1(\tau=n) \\
& \leqslant f\left(V_{\tau}\right)\left(1+\sum_{n \geqslant 1} E_{n}\left\{\left|x_{n}\right|^{2} \cdot 1\left(\left|x_{n}\right|^{2}>f\left(V_{n}\right)\right) / f\left(V_{n}\right)\right\}\right) .
\end{aligned}
$$


Hence, the result follows from (1.4) and Markov's inequality.

We put $g(x)=x / f(x)$ and define for $k \geqslant 1$

$$
t_{k}=g^{-1}\left(k^{25 d}\right), \quad h_{k}=t_{k}-t_{k-1} .
$$

REMARK. In $\$ 3$ we need to assume that the dimension $d$ may increase with $k$. (See (3.1) below.) Accordingly, all the lemmas that follow are proved valid for all $d \ll \log k$.

LEMMA 2.2. We have

$$
t_{k} \ll k h_{k}, \quad h_{k} \ll k^{-\varepsilon} t_{k}
$$

where $\varepsilon>\frac{1}{2}$.

Proof. Because $f(x)=x / g(x)$ is nondecreasing,

$$
g(x) / g^{-1}(g(x)) \geqslant g(y) / g^{-1}(g(y)) \Leftrightarrow x \leqslant y \Leftrightarrow g(x) \leqslant g(y)
$$

since also $g$ is increasing. Thus,

$$
s / g^{-1}(s) \geqslant t / g^{-1}(t), \quad 1<s \leqslant t .
$$

Consequently,

$$
\frac{h_{k}}{t_{k}}=1-\frac{g^{-1}\left((k-1)^{25 d}\right)}{g^{-1}\left(k^{25 d}\right)} \geqslant 1-\left(\frac{k-1}{k}\right)^{25 d} \gg \frac{1}{k} .
$$

This proves half of the lemma. To prove the second half, observe that $g(x)^{1 / \alpha}-\log x$ is nondecreasing. So,

$$
\exp \left(g(x)^{1 / \alpha}\right) / g^{-1}(g(x)) \leqslant \exp \left(g(y)^{1 / \alpha}\right) / g^{-1}(g(y)) \Leftrightarrow x \leqslant y .
$$

Thus,

$$
\exp \left(s^{1 / \alpha}-t^{1 / \alpha}\right) \leqslant g^{-1}(s) / g^{-1}(t), \quad 1<s \leqslant t .
$$

This implies the second half of the lemma in the same way as before.

We set $\tau(0)=0$ and define $\tau(k)$ inductively by

$$
\tau(k)=\min \left\{\tau \geqslant \tau(k-1): V_{\tau} \geqslant t_{k}\right\}, \quad k \geqslant 1 .
$$

Then $\tau(k)-1$ is a stopping time relative to $\left\{\mathcal{L}_{n}, n \geqslant 1\right\}$.

LEMMA 2.3. As $k \rightarrow \infty$

$$
P\left\{V_{\tau(k)-1} \leqslant \frac{1}{2} t_{k}\right\} \leqslant k^{-5 d}
$$

and

$$
P\left\{\left|h_{k}-\left(V_{\tau(k)-1}-V_{\tau(k-1)-1}\right)\right| \geqslant k^{-20 d}\right\} \ll k^{-5 d} .
$$

Proof. From (1.1), (1.2) and (2.1),

$$
\operatorname{tr}\left(\sigma_{\tau(k)}\right)=V_{\tau(k)}-V_{\tau(k)-1}
$$

Substituting this in Lemma 2.1 we get

$$
V_{\tau(k)-1} \geqslant V_{\tau(k)}-k^{5 d} f\left(V_{\tau(k)}\right)
$$


except on a set of probability $\ll k^{-5 d}$. Thus, aside from this small set, (2.1) and (2.2) yield that

$$
t_{k} \geqslant V_{\tau(k)-1} \geqslant t_{k}\left(1-k^{5 d} / g\left(t_{k}\right)\right) \geqslant t_{k}\left(1-k^{-20 d}\right) .
$$

The lemma follows now via easy calculations.

2.2. The skeleton process. Recalling (1.3), (2.1) and (2.2) we define

$$
x_{n k}=h_{k}^{-1 / 2} x_{n} \cdot 1(\tau(k-1) \leqslant n<\tau(k)), \quad z_{n k}^{*}=x_{n k} \cdot 1\left(\left|x_{n}\right|^{2} \leqslant f\left(V_{n}\right)\right) .
$$

Center $z_{n k}^{*}$ at its conditional expectation by writing

$$
z_{n k}=z_{n k}^{*}-E_{n} z_{n k}^{*}, \quad k, n \geqslant 1 .
$$

LEMMA 2.4. As $M \rightarrow \infty$

$$
\sum_{k \leqslant M+1} \sum_{n \geqslant 1} h_{k}^{1 / 2}\left|z_{n k}-z_{n k}^{*}\right| \ll t_{M}^{1 / 2} g\left(t_{M}\right)^{-1 / 3} \text { a.s. }
$$

Proof. The expectation of the inner sum does not exceed

$$
\begin{gathered}
\sum_{n \geqslant 1} E\left|E_{n}\left(h_{k}^{1 / 2} z_{n k}^{*}\right)\right| \leqslant \sum_{n \geqslant 1} E\left\{\left|x_{n}\right| \cdot 1\left(\left|x_{n}\right|^{2}>f\left(V_{n}\right)\right) \cdot 1(\tau(k-1) \leqslant n<\tau(k))\right\} \\
\leqslant f^{1 / 2}\left(t_{k}\right) \sum_{n \geqslant 1} E\left\{\left|x_{n}\right|^{2} \cdot 1\left(\left|x_{n}\right|^{2}>f(V)\right) / f\left(V_{n}\right)\right\}=D \cdot f^{1 / 2}\left(t_{k}\right) .
\end{gathered}
$$

Therefore, as $f$ is nondecreasing,

$$
E\left(\sum_{k \leqslant M+1} \sum_{n \geqslant 1} h_{k}^{1 / 2}\left|z_{n k}-z_{n k}^{*}\right|\right) \ll \sum_{k \leqslant M+1} f^{1 / 2}\left(t_{k}\right) \ll M \cdot f^{1 / 2}\left(t_{M+1}\right) .
$$

With an application of the Borel Cantelli lemma we thus bound the order of magnitude of the total sum by

$$
M^{3} f^{1 / 2}\left(t_{M+1}\right) \text { a.s. }
$$

Finally, using (2.2) and Lemma 2.2,

$$
M^{3} f^{1 / 2}\left(t_{M+1}\right) \ll t_{M+1}^{1 / 2} g\left(t_{M}\right)^{-1 / 3} \ll t_{M}^{1 / 2} g\left(t_{M}\right)^{-1 / 3} .
$$

LEMMA 2.5.

$$
P\left\{x_{n k} \neq z_{n k}^{*} \text { for infinitely many pairs }(n, k)\right\}=0 .
$$

Proof. Evidently $\Sigma_{k, n \geq 1} P\left\{x_{n k} \neq z_{n k}^{*}\right\} \leqslant D$. Hence, the Borel Cantelli lemma implies the result.

We put

$$
Z_{n k}=\sum_{\nu \leqslant n} z_{\nu k}, \quad Z_{k}=Z_{\infty k}, \quad \mathscr{F}_{k}=\mathscr{L}_{\tau(k)-1}
$$

and

$$
\Gamma_{n k}=\sum_{\nu \leqslant n} \gamma_{\nu k}, \quad \Gamma_{k}=\Gamma_{\infty k}
$$

where $\gamma_{n k}=\left(\left(\gamma_{n k}(i, j)\right), 1 \leqslant i, j \leqslant d\right)$ is the matrix with entries

$$
\gamma_{n k}(i, j)=E_{n}\left\{\left\langle z_{n k}, e_{i}\right\rangle\left\langle z_{n k}, e_{j}\right\rangle\right\}, k, n \geqslant 1 \text {. }
$$


We call the process $\left\{\sum_{k \leqslant n} h_{k}^{1 / 2} Z_{k}, \mathscr{F}_{n} ; n \geqslant 1\right\}$ the skeleton process of the given martingale. In the sequel we approximate this process by $\left\{X\left(t_{n}\right), n \geqslant 1\right\}$ which is the corresponding skeleton process for the Brownian motion. (See (2.20) below.) To estimate the gaps left by the skeleton process of the martingale we need the following lemma.

LEMMA 2.6. As $k \rightarrow \infty$

$$
\sup _{n \geqslant 1} h_{k}^{1 / 2}\left|Z_{n k}\right| \ll t_{k}^{1 / 2}\left(g\left(t_{k}\right)\right)^{-1 /(50 d)} \text { a.s. }
$$

Proof. In view of Lemma 2.2, it is enough to show

$$
\sum_{k \geqslant 1} P\left\{\sup _{n \geqslant 1} h_{k}^{1 / 2}\left\langle u, Z_{n k}\right\rangle>\left(\frac{1}{d}\right) h_{k}^{1 / 2} \log ^{3} k\right\}<\infty
$$

for any $u \in \mathbf{R}^{d}$ with $|u|=1$. Define

$$
W_{n}=h_{k}^{1 / 2}\left\langle u, Z_{n k}\right\rangle, \quad \lambda=h_{k}^{-1 / 2}, \quad c=2 f^{1 / 2}\left(t_{k}\right)
$$

and

$$
S_{n}=\exp \left(\lambda W_{n}-\frac{1}{2} \lambda^{2}\left(1+\frac{1}{2} \lambda c\right) h_{k} \sum_{\nu \leqslant n}\left\langle u, \gamma_{\nu k} u\right\rangle\right)
$$

Then, by Lemma 2.2, (2.2)-(2.8), Lemma 5.4.1 and Corollary 5.4.1 of Stout [14] and our choice of $c$, the sequence $\left\{S_{n}, \mathscr{L}_{n} ; n \geqslant 1\right\}$ is a supermartingale satisfying

$$
P\left\{\sup _{n \geqslant 1} S_{n}>\alpha\right\}<1 / \alpha
$$

for each $\alpha>0$ and sufficiently large $k$. Putting now $\alpha=k^{2}$, we obtain

$$
P\left\{\sup _{n \geqslant 1}\left(\lambda W_{n}-\frac{1}{2}(1+\lambda c)\left\langle u, \Gamma_{n k} u\right\rangle\right) \geqslant 2 \log k\right\} \ll k^{-2} .
$$

Hence, since $\left\langle u, \Gamma_{n k} u\right\rangle \leqslant\left\langle u, \Gamma_{k} u\right\rangle$ for all $u$ and all $n \geqslant 1$,

$$
P\left\{\sup _{n \geqslant 1} \lambda W_{n}>\left(\frac{1}{d}\right) \log ^{3} k\right\} \ll P\left\{\sup _{|u|=1}\left\langle u, \Gamma_{k} u\right\rangle>2 \log k\right\}+k^{-2} \ll k^{-2} .
$$

In view of (2.8), this concludes the proof of the lemma.

2.3. A conditional central limit theorem. For the proof of our theorem, we will eventually apply Theorem 1 of [1] to the sequence $\left\{Z_{k}, \mathscr{F}_{k} ; k \geqslant 1\right\}$. To this end we shall establish the following proposition. Denote $E\left(\cdot \mid \mathscr{F}_{k-1}\right)$ by $E_{k}^{*}(\cdot)$.

Proposition 1. As $k \rightarrow \infty$

$$
\sup _{u \in \mathbf{R}^{d},|u| \leqslant k^{2}} E\left|E_{k}^{*}\left\{\exp \left(i\left\langle u, Z_{k}\right\rangle\right)\right\}-\exp \left(-\frac{1}{2}\langle u, A u\rangle\right)\right| \ll k^{-5 d} .
$$

For the proof of Proposition 1 we modify a method of Brown [2]. (See also Gänssler and Stute [6, Chapter 9].) We divide the argument into several steps. These are formulated as lemmas. 
LEMMA 2.7. There is a constant $C$ such that for all $k, l \geqslant 1$

$$
P\left\{\sup _{u \in \mathbf{R}^{d},|u| \leqslant k^{2}}\left\langle u,\left(\Gamma_{k}-A\right) u\right\rangle \geqslant C k^{-5 d} l\right\} \leqslant e^{-k l}
$$

and

$$
P\left\{\inf _{u \in \mathbf{R}^{d},|u| \leqslant k^{2}}\left\langle u,\left(\Gamma_{k}-A\right) u\right\rangle \leqslant-C k^{-5 d}\right\} \ll k^{-5 d} .
$$

Proof. To prove the first assertion, we find by (1.5), (2.4), (2.5), (2.7), (2.8) and elementary calculations that

$$
\begin{aligned}
\left\langle u,\left(\Gamma_{k}-A\right) u\right\rangle \leqslant & h_{k}^{-1}\left\langle u,\left(A_{\tau(k)-1}-A_{\tau(k-1)}\right) u\right\rangle+h_{k}^{-1}|u|^{2} \cdot f\left(V_{\tau(k-1)}\right)-\langle u, A u\rangle \\
\leqslant & h_{k}^{-1}\langle u, A u\rangle\left(V_{\tau(k)-1}-V_{\tau(k-1)}-h_{k}\right) \\
& +h_{k}^{-1}|u|^{2}\left(1+2 \sup _{n} \frac{\left\|A_{n}-V_{n} A\right\|}{f\left(V_{n}\right)}\right) f\left(V_{\tau(k-1)}\right) .
\end{aligned}
$$

Moreover, if $\tau(k-1)=\tau(k)$ then $\left\langle u,\left(\Gamma_{k}-A\right) u\right\rangle \leqslant 0$. Hence, (2.2), (2.3) and Lemma 2.2 provide that

$$
\sup _{u \in \mathbf{R}^{d},|u| \leqslant k^{2}}\left\langle u,\left(\Gamma_{k}-A\right) u\right\rangle \leqslant k^{-20 d}\left(1+\sup _{n} \frac{\left\|A_{n}-V_{n} A\right\|}{f\left(V_{n}\right)}\right) .
$$

Thus we obtain (2.9) using (1.5) and Markov's inequality. Combining similar calculations with the idea of the proof of Lemma 2.1, (2.10) follows from (1.4), (1.5), Markov's inequality and Lemma 2.3.

We shall use the following facts in the same context as did Brown [2].

LEMMA 2.8. Let $Q(0)=0$ and

$$
\begin{aligned}
& Q(t)=2 t^{-2}\left(e^{i t}-1-i t+\frac{1}{2} t^{2}\right), \quad t \neq 0, \\
& M(t)=\min (2, t / 3)
\end{aligned}
$$

and

$$
N(t)=e^{-t}-1+t
$$

Then

$$
\begin{aligned}
& |1-Q(t)| \leqslant 1, \quad|Q(t)| \leqslant M(|t|), \quad t \in \mathbf{R}, \\
& |N(t)| \leqslant \frac{1}{2} t^{2}, \quad t \geqslant 0
\end{aligned}
$$

and

$$
\left|e^{t}-1\right| \leqslant 2|t|, \quad t \leqslant \frac{1}{2}
$$


We now break in two the quantity being estimated in Proposition 1. We obtain

$$
\begin{aligned}
&\left|E_{k}^{*}\left\{\exp \left(i\left\langle u, Z_{k}\right\rangle\right)\right\}-\exp \left(-\frac{1}{2}\langle u, A u\rangle\right)\right| \\
& \leqslant\left|E_{k}^{*}\left\{\left(1-\exp \left(\frac{1}{2}\left\langle u,\left(\Gamma_{k}-A\right) u\right\rangle\right)\right) \exp \left(i\left\langle u, Z_{k}\right\rangle\right)\right\}\right| \\
&+\left|\exp \left(-\frac{1}{2}\langle u, A u\rangle\right)-E_{k}^{*}\left\{\exp \left(\frac{1}{2}\left\langle u,\left(\Gamma_{k}-A\right) u\right\rangle+i\left\langle u, Z_{k}\right\rangle\right)\right\}\right| \\
& \leqslant E_{k}^{*}\left|1-\exp \left(\frac{1}{2}\left\langle u,\left(\Gamma_{k}-A\right) u\right\rangle\right)\right| \\
&+\exp \left(-\frac{1}{2}\langle u, A u\rangle\right)\left|E_{k}^{*}\left\{1-\exp \left(i\left\langle u, Z_{k}\right\rangle+\frac{1}{2}\left\langle u, \Gamma_{k} u\right\rangle\right)\right\}\right| \\
&= \text { I }+ \text { II } \text { (say). }
\end{aligned}
$$

Next, the expectations of these expressions are estimated.

LEMMA 2.9. As $k \rightarrow \infty$

$$
\sup _{u \in \mathbf{R}^{d},|u| \leqslant k^{2}} E \mathrm{I} \ll k^{-5 d} .
$$

Proof. Put $F_{k}=\left\{\sup _{|u| \leqslant k^{2}}\left|\left\langle u,\left(\Gamma_{k}-A\right) u\right\rangle\right| \geqslant C k^{-5 d}\right\}$ where the constant $C$ appears in Lemma 2.4. Then by Lemmas $2.4,2.5$ and 2.8 we have for all $|u| \leqslant k^{2}$

$$
\begin{aligned}
E(\mathrm{I}) & =\left(\int_{F_{k}}+\int_{F_{k}^{c}}\right)\left|1-\exp \left(\frac{1}{2}\left\langle u,\left(\Gamma_{k}-A\right) u\right\rangle\right)\right| d P \\
& \ll P\left(F_{k}\right)+\sum_{l \geqslant 1} \exp \left(C k^{-5 d} l-k l\right)+k^{-5 d} \ll k^{-5 d}
\end{aligned}
$$

To estimate $E$ II we introduce the random variables

$$
Y_{n k}=\exp \left(i\left\langle u, Z_{n-1, k}\right\rangle+\frac{1}{2}\left\langle u, \Gamma_{n k} u\right\rangle\right)\left\{\exp \left(i\left\langle u, z_{n k}\right\rangle\right)-\exp \left(-\frac{1}{2}\left\langle u, \gamma_{n k} u\right\rangle\right)\right\} .
$$

Here we have used notation introduced in (2.4)-(2.7). Thus,

$$
\sum_{n \geqslant 1} Y_{n k}=\exp \left(i\left\langle u, Z_{k}\right\rangle+\frac{1}{2}\left\langle u, \Gamma_{k} u\right\rangle\right)-1 \text {. }
$$

Furthermore, by (2.7), (2.12), and (2.9),

$$
E \sup _{n \geqslant 1} \sup _{|u| \leqslant k^{2}}\left|\sum_{\nu \leqslant n} Y_{\nu k}\right| \leqslant 1+E \sup _{|u| \leqslant k^{2}} \exp \left(\frac{1}{2}\left\langle u, \Gamma_{k} u\right\rangle\right)<\infty .
$$

Finally, by (2.2)-(2.7) and (2.12) one has that $Y_{n k}$ and $E_{n}\left(Y_{n k}\right)$ are both zero unless $\tau(k-1) \leqslant n<\tau(k)$. Consequently,

$$
E_{k}^{*} Y_{n k}=E_{k}^{*}\left(E_{n} Y_{n k}\right) \quad \text { a.s. }
$$

Lemma 2.10. As $k \rightarrow \infty$

$$
\sup _{u \in \mathbf{R}^{d},|u| \leqslant k^{2}} E \mathrm{II} \ll k^{-5 d} .
$$


Proof. Using (2.13)-(2.15), Lebesgue's dominated convergence theorem and Jensen's inequality, one gets

$$
\begin{aligned}
\exp \left(\frac{1}{2}\langle u, A u\rangle\right) E \mathrm{II} & =E\left|E_{k}^{*}\left(\sum_{n \geqslant 1} Y_{n k}\right)\right| \\
& \leqslant E\left(\sum_{n \geqslant 1}\left|E_{k}^{*}\left(E_{n} Y_{n k}\right)\right|\right) \leqslant \sum_{n \geqslant 1} E\left|E_{n}\left(Y_{n k}\right)\right|
\end{aligned}
$$

Thus our plan is to first estimate $E_{n} Y_{n k}$. Applying its definition (2.12) and the notation of Lemma 2.8, we can rewrite $Y_{n k}$ in the form:

$$
\begin{aligned}
Y_{n k} & =\exp \left(i\left\langle u, Z_{n k}\right\rangle+\frac{1}{2}\left\langle u, \Gamma_{n k} u\right\rangle\right) \\
& \times\left\{i\left\langle u, z_{n k}\right\rangle-\frac{1}{2}\left\langle u, z_{n k}\right\rangle^{2}\left(1-Q\left(\left\langle u, z_{n k}\right\rangle\right)\right)-N\left(\frac{1}{2}\left\langle u, \gamma_{n k} u\right\rangle\right)+\frac{1}{2}\left\langle u, \gamma_{n k} u\right\rangle\right\}
\end{aligned}
$$

Hence,

$$
\begin{aligned}
\left|E_{n} Y_{n k}\right| \exp ( & \left.-\frac{1}{2}\left\langle u, \Gamma_{n k} u\right\rangle\right) \\
& \leqslant \frac{1}{2} E_{n}\left\{\left\langle u, z_{n k}\right\rangle^{2}\left|Q\left(\left\langle u, z_{n k}\right\rangle\right)\right|\right\}+E_{n}\left|N\left(\frac{1}{2}\left\langle u, \gamma_{n k} u\right\rangle\right)\right| .
\end{aligned}
$$

By (2.1)-(2.5), (2.7), Lemmas 2.2 and 2.8 and Cauchy's inequality the right-hand side of (2.17) is bounded for all $|u| \leqslant k^{2}$ by

$$
\begin{aligned}
& \frac{1}{2} E_{n}\left(\left\langle u, z_{n k}\right\rangle^{2}\right) M\left(\left|\left\langle u, z_{n k}\right\rangle\right|\right)+\frac{1}{8}\left\langle u, \gamma_{n k} u\right\rangle^{2} \\
& \leqslant E_{n}\left\{\left\langle u, z_{n k}\right\rangle^{2} \cdot \frac{1}{3}|u| h_{k}^{-1 / 2}\left(f^{1 / 2}\left(V_{\tau(k)-1}\right)+E_{n}\left(f^{1 / 2}\left(V_{\tau(k)-1}\right)\right)\right)\right\} \\
& \quad+\frac{1}{8}\left\langle u, \gamma_{n k} u\right\rangle^{2} \\
& \leqslant\left\langle u, \gamma_{n k} u\right\rangle\left\{\sup _{n}\left\langle u, \gamma_{n k} u\right\rangle+|u| h_{k}^{-1 / 2} f^{1 / 2}\left(t_{k}\right)\right\} \\
& \ll\left\langle u, \gamma_{n k} u\right\rangle k^{-12 d+3} .
\end{aligned}
$$

Therefore, because $\left\langle u, \Gamma_{n k} u\right\rangle \leqslant\left\langle u, \Gamma_{k} u\right\rangle$ for all $u$ and all $n \geqslant 1$,

$$
\exp \left(-\frac{1}{2}\left\langle u, \Gamma_{k} u\right\rangle\right) \sum_{n \geqslant 1}\left|E_{n} Y_{n k}\right| \ll k^{-12 d+3}\left\langle u, \Gamma_{k} u\right\rangle,|u| \leqslant k^{2} .
$$

Consequently, by (2.9),

$$
\begin{aligned}
\sup _{u \in \mathbf{R}^{d},|u| \leqslant k^{2}} & \exp \left(-\frac{1}{2}\langle u, A u\rangle\right) \sum_{n \geqslant 1} E\left|E_{n} Y_{n k}\right| \\
& \ll k^{-12 d+3} E\left\{\sup _{|u| \leqslant k^{2}}\left(\left\langle u,\left(\Gamma_{k}-A\right) u\right\rangle+\langle u, A u\rangle\right) \cdot \exp \left(\frac{1}{2}\left\langle u,\left(\Gamma_{k}-A\right) u\right\rangle\right)\right\} \\
& \ll k^{-5 d} .
\end{aligned}
$$

Comparing this with (2.16), we find the proof complete.

Proposition 1 follows from Lemmas 2.9, 2.10 and (2.11).

2.4. Completion of the proof of Theorem $1(d<\infty)$. We apply Theorem 1 of [1] with $T_{k}=k^{5 / 4}$ in conjunction with Proposition 1 . Hence without changing its 
distribution we can redefine the sequence $\left\{Z_{k}, k \geqslant 1\right\}$ on a (possibly) richer probability space on which there exists a sequence $\left\{U_{k}, k \geqslant 1\right\}$ of independent random vectors each having Gaussian distribution $N(0, A)$ such that

$$
P\left(\left|Z_{k}-U_{k}\right| \geqslant \alpha_{k}\right) \leqslant \alpha_{k}
$$

where

$$
\alpha_{k} \ll\left(k^{-5 d}\right)^{1 / 2}\left(k^{5 / 4}\right)^{d}+k^{-5 / 4} \log k+P\left(|N(0, A)| \geqslant \frac{1}{4} k^{5 / 4}\right) \ll k^{-9 / 8} .
$$

Consequently, by the Borel Cantelli lemma we have

$$
\left|Z_{k}-U_{k}\right| \ll k^{-9 / 8} \text { a.s. }
$$

We now apply twice Lemma Al of [1]. In the first application we choose $S_{1}=S_{2}=S_{3}=\left(\mathbf{R}^{d}\right)^{\infty}$ and $F$ the joint distribution of the martingale difference sequence $\left\{x_{n}, n \geqslant 1\right\}$ and $\left\{Z_{k}, k \geqslant 1\right\}$ and $G$ the joint distribution of the (redefined) sequence $\left\{Z_{k}, k \geqslant 1\right\}$ and $\left\{U_{k}, k \geqslant 1\right\}$. In the second application we choose $S_{1}=$ $\left(\mathbf{R}^{d}\right)^{\infty}, S_{2}=\left(\mathbf{R}^{d}\right)^{M}$ and $S_{3}=C_{\mathbf{R}^{d}}\left[0, t_{M}\right]$ for fixed $M \in \mathbf{Z}^{+}$and $F$ the joint distribution of the (redefined) sequences $\left\{x_{n}, n \geqslant 1\right\}$ and $\left\{U_{k}, k \geqslant 1\right\}$ and $G$ the joint distribution of $\left(X\left(t_{k}\right)\right)-\left\{X\left(t_{k-1}\right), 1 \leqslant k \leqslant M\right\}$ and $\left\{X(t), 0 \leqslant t \leqslant t_{M}\right\}$. By the conclusion of Lemma Al of [1] we obtain a consistent system of distributions as $M$ ranges through $\mathbf{Z}^{+}$.

Thus by Kolmogorov's theorem, the above remarks, (2.19), (2.1), (2.5) and Lemma 2.2 we have, with probability 1 ,

$$
\begin{aligned}
\sum_{k \leqslant M} \sum_{n \geqslant 1} h_{k}^{1 / 2} z_{n k}-X\left(t_{M}\right) & \ll \sum_{k \leqslant M} h_{k}^{1 / 2} k^{-9 / 8} \ll \sum_{k \leqslant M} t_{k}^{1 / 2} g\left(t_{k}\right)^{-3 / 50 d} \\
& \ll t_{M}^{1 / 2} g\left(t_{M}\right)^{-1 / 50 d} .
\end{aligned}
$$

Further, elementary considerations yield:

$$
\sup _{t_{M} \leqslant t \leqslant t_{M+1}}\left|X(t)-X\left(t_{M-1}\right)\right| \ll t_{M}^{1 / 2} g\left(t_{M}\right)^{-1 / 50 d} \text { a.s. }
$$

(For details see for instance, pp. 43 and 44 of [1].) Finally let $t \geqslant 1$ be given. This point determines $M$ with $t_{M} \leqslant t<t_{M+1}$. Then by (2.19), (2.20) and Lemmas 2.4-2.6

$$
\begin{aligned}
\mid \sum_{n \geqslant 1} x_{n} \cdot 1\left(V_{n} \leqslant\right. & t)-X(t)\left|\leqslant h_{M+1}^{1 / 2} \sup _{n \geqslant 1}\right| Z_{n, M+1}\left|+\sum_{k \leqslant M+1} h_{k}^{1 / 2} \sum_{n \geqslant 1}\right| z_{n k}-z_{n k}^{*} \mid \\
& +\left|\sum_{k \leqslant M} \sum_{n \geqslant 1} h_{k}^{1 / 2} z_{n k}-X\left(t_{M}\right)\right|+\sup _{t_{M} \leqslant t \leqslant t_{M+1}}\left|X(t)-X\left(t_{M}\right)\right|+0(1) \\
\ll & t_{M}^{1 / 2} g\left(t_{M}\right)^{-1 /(50 d)} \ll t^{1 / 2} g(t)^{-1 /(50 d)} \ll t^{1 / 2}(f(t) / t)^{1 /(50 d)} \text { a.s. }
\end{aligned}
$$

3. Proof of Theorem $1(d=\infty)$. Our plan is to approximate the difference sequence of the skeleton process by finite-dimensional random variables. The dimension $d_{k}$ of the approximating $k$ th difference will be allowed to tend to infinity at a slow rate. As indicated in the remark preceding Lemma 2.2, we can thereby use much of the material developed in $\$ 2$. But, of course, at some point we must treat the difference between the given martingale and its approximation. In essence, our success hinges on a proof of a bounded law on the iterated logarithm for $H$-valued 
martingales. This is given in $§ 3.2$. Besides the properly modified versions of the lemmas of $\S 2$ we need several more lemmas, some of which will contribute directly to the final estimate (3.27) below.

Recalling the definition of $g(x)=x / f(x)$ and the hypotheses on $f$ we conclude that for some $\alpha(x) \uparrow \infty$

$$
g(x)=\exp (-\alpha(x) \log \log x) .
$$

Because $\max \left\{f(x), x \exp \left(-(\log \log x)^{2}\right)\right\}$ still satisfies the hypotheses of the theorem, we can assume without loss of generality that

$$
\alpha(x) \leqslant \log \log x .
$$

We define for $k \geqslant 1$

$$
t_{k}=\exp \left(k^{1 / 4}\right), \quad h_{k}=t_{k}-t_{k-1} \sim \frac{1}{4} k^{-3 / 4} t_{k}, \quad d_{k}=\alpha\left(t_{k}\right) / 100 .
$$

Then

$$
f\left(t_{k}\right)=t_{k} / g\left(t_{k}\right) \leqslant t_{k} k^{-25 d_{k}}
$$

Next, we define a sequence $\left\{\pi_{k} ; k \geqslant 1\right\}$ of mappings $\pi_{k}: H \rightarrow H$ by

$$
\pi_{k} x=\sum_{i \leqslant d_{k}}\left\langle x, e_{i}\right\rangle e_{i}, \quad x \in H
$$

Using the notation introduced in $\$ 2$ but including the appropriate changes imposed by (3.1), we write

$$
z_{n k}^{\prime}=\pi_{k} z_{n k}, \quad Z_{n k}^{\prime}=\pi_{k} Z_{n k}=\sum_{\nu \leqslant n} z_{\nu k}^{\prime}, \quad Z_{k}^{\prime}=Z_{\infty k}^{\prime} .
$$

Remark (3.4). Proposition 1 remains valid with $Z_{k}^{\prime}$ and $d_{k}$ in place of $Z_{k}$ and $d$ respectively.

This is immediate from its proof since by (3.1) $d_{k} \ll \log k$.

3.1. Maximal inequalities. We need a minor extension of Burkholder's square function inequality for martingales.

LEMMA 3.1. Let $\left\{\varphi_{n}, \mathcal{G}_{n} ; n \geqslant 1\right\}$ be a martingale with values in a separable Hilbert space. Let $\mathcal{G}$ be a sub- $\sigma$-field of $\mathcal{G}_{1}$. Then for every $1 \leqslant p<\infty$ there are constants $c_{p}$ and $C_{p}$ such that

$$
\begin{aligned}
c_{p} E_{\mathcal{G}}\left(\sup _{n}\left|\varphi_{n}\right|^{p}\right) & \leqslant E_{\mathcal{G}}\left(\left(\left|\varphi_{1}\right|^{2}+\sum_{n \geqslant 1}\left|\varphi_{n+1}-\varphi_{n}\right|^{2}\right)^{p / 2}\right) \\
& \leqslant C_{P} E_{\mathcal{S}}\left(\sup _{n}\left|\varphi_{n}\right|^{p}\right) .
\end{aligned}
$$

Here $E_{\mathcal{G}}$ denotes the conditional expectation operator with respect to $\mathcal{G}$.

Proof. If $\mathcal{G}$ is the trivial $\sigma$-field, the lemma is well known (see e.g. [3, Theorem 3.2]). For general $\mathcal{G}$, let $\Lambda \in \mathcal{G}$ and apply this special case to the martingale $\left\{\varphi_{n} \cdot 1_{\Lambda}, \mathcal{G}_{n} ; n \geqslant 1\right\}$. The lemma follows then by elementary arguments involving conditional expectations. 
LEMMA 3.2. As $k \rightarrow \infty$ we have

$$
\left\|E_{k}^{*}\left\{\sup _{n \geqslant 1}\left|Z_{n k}\right|^{4}\right\}\right\|_{\infty} \ll 1
$$

Proof. Let

$$
\xi_{n}=x_{n} \cdot 1\left(\left|x_{n}\right|^{2} \leqslant f\left(V_{n}\right)\right), \quad \chi_{n k}=1(\tau(k-1) \leqslant n<\tau(k)) .
$$

We apply Lemma 3.1 with $p=4$ and $\mathcal{G}=\mathscr{F}_{k-1}$ to the martingale $\left\{h_{k}^{1 / 2} Z_{n k}, \mathscr{L}_{n} \vee\right.$ $\left.\mathscr{F}_{k-1} ; n \geqslant 1\right\}$ and obtain using (2.4), (2.5), and (3.6),

$$
\begin{aligned}
E_{k}^{*}\left\{\sup _{n} h_{k}^{2}\left|Z_{n k}\right|^{4}\right\} \ll E_{k}^{*}\left\{\left(\sum_{n \geqslant 1}\left|\xi_{n}-E_{n} \xi_{n}\right|^{2} \chi_{n k}\right)^{2}\right\} \\
\ll E_{k}^{*}\left\{\sum_{n \geqslant 1}\left(\left|\xi_{n}\right|^{2}+E_{n}\left(\left|\xi_{n}\right|^{2}\right)\right)^{2} \chi_{n k}\right\} \\
+E_{k}^{*}\left\{\sum_{n \geqslant 1}\left(\left|\xi_{n}\right|^{2}+E_{n}\left(\left|\xi_{n}\right|^{2}\right)\right) \chi_{n k} \sum_{1 \leqslant m<n}\left(\left|\xi_{n}\right|^{2}+E_{m}\left(\left|\xi_{m}\right|^{2}\right)\right) \chi_{m k}\right\} \\
=\mathrm{I}+\mathrm{II} \text { (say). }
\end{aligned}
$$

To treat I, we estimate one factor $\left|\xi_{n}\right|^{2}+E_{n}\left(\left|\xi_{n}\right|^{2}\right)$ by $f\left(t_{k}\right)$. This can be done in light of (3.5) and (2.3). Thus, by (3.1), (3.2) and (2.1),

$$
\begin{aligned}
\mathrm{I} & \ll f\left(t_{k}\right) E_{k}^{*}\left\{\sum_{n \geqslant 1} \chi_{n k} E_{n}\left(\left|\xi_{n}\right|^{2}\right)\right\} \\
& \ll f\left(t_{k}\right)\left(V_{\tau(k)-1}-V_{\tau(k-1)}+E_{k}^{*}\left(\left|\xi_{\tau(k-1)}\right|^{2}\right)\right) \\
& \ll f\left(t_{k}\right)\left(h_{k}+f\left(t_{k}\right)\right) \ll h_{k}^{2} .
\end{aligned}
$$

Similarly,

$$
\begin{aligned}
\mathrm{II} & \ll E_{k}^{*}\left\{\sum_{n \geqslant 1} \chi_{n k} E_{n}\left(\left|\xi_{n}\right|^{2}\right) \cdot \sum_{1 \leqslant m<n} \chi_{m k}\left(\left|\xi_{m}\right|^{2}+E_{m}\left(\left|\xi_{m}\right|^{2}\right)\right)\right\} \\
& \ll\left(h_{k}+f\left(t_{k}\right)\right) E_{k}^{*}\left\{\sum_{m \geqslant 1} \chi_{m k}\left(\left|\xi_{m}\right|^{2}+E_{m}\left(\left|\xi_{m}\right|^{2}\right)\right)\right\} \\
& \ll h_{k} E_{k}^{*}\left\{\sum_{m \geqslant 1} \chi_{m k} E_{m}\left(\left|\xi_{m}\right|^{2}\right)\right\} \ll h_{k}^{2} .
\end{aligned}
$$

The next lemma is an infinite-dimensional analogue of Lemma 2.6.

LEMMA 3.3. As $k \rightarrow \infty$ we have with probability 1

$$
\sup _{n \geqslant 1} h_{k}^{1 / 2}\left|Z_{n k}\right| \ll t_{k}^{1 / 2} \text {. }
$$

Proof. This follows at once from Markov's inequality, Lemma 3.2 and the Borel Cantelli lemma since, by (3.1), $\Sigma_{k \geqslant 1} h_{k}^{2} t_{k}^{-2} \ll \Sigma_{k \geqslant 1} k^{-3 / 2}<\infty$.

The next lemma serves the same purpose as the preceding one. Namely, it gives us a bound on the fluctuation of the Brownian motion within the gaps left by its skeleton process. It is an easy consequence of Fernique's theorem [5] and the Borel Cantelli lemma. 
Lemma 3.4. As $k \rightarrow \infty$ we have for any Brownian motion $\{X(t) ; t \geqslant 0\}$ with mean 0 and covariance matrix $A$

$$
\sup _{t_{k-1} \leqslant t \leqslant t_{k}}\left|X(t)-X\left(t_{k}\right)\right| \ll t_{k}^{1 / 2} \quad \text { a.s. }
$$

3.2. Exponential inequalities. Put $Q_{k}=I-\pi_{k}$ where $I$ is the identity map and $\pi_{k}$ is defined in (3.3).

Proposition 2. As $m \rightarrow \infty$ we have with probability 1

$$
\left|\sum_{k \leqslant m} h_{k}^{1 / 2} Q_{k}\left(Z_{k}\right)\right|=o\left(\left(t_{m} \log \log t_{m}\right)^{1 / 2}\right) .
$$

As mentioned in the introductory remarks of this section, Proposition 2 amounts to a bounded law of the iterated logarithm. As a rule these are proved by establishing exponential bounds. Unfortunately for Banach spaces, such bounds are available only in the presence of certain dependence structures, like those embodied by $\varphi$-mixing or absolutely regular sequences (see [9, Proposition 3.1$]$ and $[4, \S 8]$ ). For martingales and strongly mixing random variables as well, only the Hilbert space case can be treated so far. This treatment is accomplished by a device, due to Goodman, Kuelbs and Zinn [7, p. 731] which will allow us to reduce considerations for $H$-valued martingales to the study of real-valued martingales. (For the strong mixing case, see [4].) We will need a variant of one of their lemmas for use in the same context. Its proof is left to the reader.

LEMMA 3.5. Let $\rho>0$ and let $\left\{a_{k}, k \geqslant 1\right\},\left\{b_{k}, k \geqslant 1\right\}$ be sequences of real numbers with $a_{k} \geqslant 1$ and $b_{k}>0$ for all $k$. Put $A_{m}=\sum_{k \leqslant m} a_{k}$. Suppose that

$$
b_{m}^{2} \leqslant \rho \sum_{k<m} a_{k} b_{k} \log \log \sum_{k<m} b_{k}
$$

for all sufficiently large $m$. Then

$$
\limsup _{m \rightarrow \infty} \frac{b_{m}}{A_{m} \log \log A_{m}} \leqslant \rho .
$$

For the proof of Proposition 2 we set

$$
u_{k}=h_{k}^{1 / 2} Q_{k}\left(Z_{k}\right), \quad k \geqslant 1 \text {. }
$$

By Lemma 3.2, the conditional version of Lebesgue's dominated convergence theorem and (2.15) (with $z_{n k}$ in place of $Y_{n k}$ ) we get

$$
E_{k}^{*} Q_{k}\left(Z_{k}\right)=\lim _{n \rightarrow \infty} E_{k}^{*} Q_{k}\left(Z_{n k}\right)=Q_{k} \lim _{n \rightarrow \infty} E_{k}^{*} Z_{n k}=0
$$

for all $k \geqslant 1$. Hence, $\left\{u_{k}, \mathscr{F}_{k} ; k \geqslant 1\right\}$ is a martingale difference sequence. Let

$$
T_{m}=\sum_{k \leqslant m} u_{k} \text {. }
$$

Then (see [7, relation $(4.28)])$

$$
\left|T_{m}\right|^{2}=\sum_{k \leqslant m}\left|u_{k}\right|^{2}+2 \sum_{k \leqslant m}\left\langle u_{k}, T_{k-1}\right\rangle .
$$


In Lemma 3.6 we prove that the first sum in (3.8) is small. The estimate of the second sum depends on the observation that it is close to a martingale to which, when properly truncated, the standard exponential bound applies.

LEMMA 3.6. As $m \rightarrow \infty$ we have with probability 1

$$
\sum_{k \leqslant m}\left|u_{k}\right|^{2} \ll t_{m}
$$

Proof. Let

$$
v_{k}=\left|u_{k}\right|^{2}-E_{k}^{*}\left(\left|u_{k}\right|^{2}\right), \quad k \geqslant 1
$$

By (3.6) and Lemma 3.2

$$
\left\|E_{k}^{*}\left(\left|u_{k}\right|^{2}\right)\right\|_{\infty} \leqslant\left\|E_{k}^{*}\left(h_{k}\left|Z_{k}\right|^{2}\right)\right\|_{\infty} \leqslant h_{k}\left\|\left(E_{k}^{*}\left(\left|Z_{k}\right|^{4}\right)\right)^{1 / 2}\right\|_{\infty} \ll h_{k} .
$$

Therefore, by (3.1) it is enough to prove:

$$
\sum_{k \leqslant m} v_{k} \ll t_{m} \text { a.s. }
$$

Now again by Lemma 3.2

$$
E\left(\left|v_{k}\right|^{2}\right) \ll E\left(\left|u_{k}\right|^{4}\right) \ll h_{k}^{2} E\left(\left|Z_{k}\right|^{4}\right) \ll h_{k}^{2} .
$$

Hence, by (3.1)

$$
\sum_{k \geqslant 1} t_{k}^{-2} E\left|v_{k}\right|^{2} \ll \sum_{k \geqslant 1} h_{k}^{-2} t_{k}^{2} \ll \sum k^{-3 / 2} \ll 1
$$

But $\left\{t_{k}^{-1} v_{k}, \mathscr{F}_{k} ; k \geqslant 1\right\}$ is a martingale difference sequence. Therefore, relation (3.9) is a consequence of the Kronecker lemma since $L^{2}$ bounded $H$-valued martingales converge almost surely.

We now consider the second sum in (3.8). Let us properly truncate the random variables $\left\langle u_{k}, T_{k-1}\right\rangle$ and center them at conditional expectations by putting

$$
w_{k}=\left(I-E_{k}^{*}\right)\left(\left\langle u_{k}, T_{k-1}\right\rangle \cdot 1\left(\left|\left\langle u_{k}, T_{k-1}\right\rangle\right| \leqslant k^{-1 / 8} t_{k}\right)\right),
$$

where $I$ denotes the identity operator. Then $\left\{w_{k}, \mathscr{F}_{k} ; k \geqslant 1\right\}$ is a martingale difference sequence. Next, we see that these alterations do not spoil things.

LEMMA 3.7. As $m \rightarrow \infty$ we have with probability 1

$$
\sum_{k \leqslant m}\left\langle u_{k}, T_{k-1}\right\rangle \cdot 1\left(\left|\left\langle u_{k}, T_{k-1}\right\rangle\right|>k^{-1 / 8} t_{k}\right) \ll t_{m}
$$

and

$$
\sum_{k \leqslant m} E_{k}^{*}\left\{\left\langle u_{k}, T_{k-1}\right\rangle \cdot 1\left(\left|\left\langle u_{k}, T_{k-1}\right\rangle\right| \leqslant k^{-1 / 8} t_{k}\right)\right\} \ll t_{m}
$$


Proof. We first estimate $E\left(\left|T_{m}\right|^{4}\right)$. By (3.6) and (3.7), $\left\{T_{m}, \mathscr{F}_{m} ; m \geqslant 1\right\}$ is a martingale. Thus, applying Lemma 3.1 with $p=4,(3.6)$ and Lemma 3.2,

$$
\begin{aligned}
E\left(\left|T_{m}\right|^{4}\right) & \ll E\left(\left(\sum_{k \leqslant m}\left|u_{k}\right|^{2}\right)^{2}\right) \\
& \ll \sum_{k \leqslant m} E\left(\left|u_{k}\right|^{4}\right)+\sum_{1 \leqslant j<k \leqslant m} E\left(\left|u_{j}\right|^{2}\left|u_{k}\right|^{2}\right) \\
& \ll \sum_{k \leqslant m} h_{k}^{2}+\sum_{1 \leqslant j<k \leqslant m} E\left\{\left|u_{j}\right|^{2} E_{k}^{*}\left(\left|u_{k}\right|^{2}\right)\right\} \\
& \ll \sum_{k \leqslant m} h_{k}^{2}+\sum_{1 \leqslant j<k \leqslant m} h_{j} h_{k} \ll t_{m}^{2} .
\end{aligned}
$$

Hence, by Lemma 3.2 and (3.1),

$$
\begin{aligned}
t_{k}^{-1} E\left(\mid\left\langle u_{k},\right.\right. & \left.\left.T_{k-1}\right\rangle \mid \cdot 1\left(\left|\left\langle u_{k}, T_{k-1}\right\rangle\right|>k^{-1 / 8} t_{k}\right)\right) \ll k^{3 / 8} t_{k}^{-4} E\left(\left|\left\langle u_{k}, T_{k-1}\right\rangle\right|^{4}\right) \\
& \ll k^{3 / 8} t_{k}^{-4} E\left(\left|u_{k}\right|^{4}\left|T_{k-1}\right|^{4}\right) \ll k^{3 / 8} t_{k}^{-4} E\left\{\left|T_{k-1}\right|^{4} E_{k}^{*}\left(\left|u_{k}\right|^{4}\right)\right\} \\
& \ll k^{3 / 8} t_{k}^{-4} h_{k}^{2} E\left(\left|T_{k-1}\right|^{4}\right) \ll k^{3 / 8} t_{k}^{-2} h_{k}^{2} \ll k^{-9 / 8}
\end{aligned}
$$

Accordingly, relation (3.11) follows from the Beppo Levi theorem and the Kronecker lemma.

To prove (3.12) we note that $E_{k}^{*}\left\langle u_{k} T_{k-1}\right\rangle=0$ since, as already observed, $\left\{T_{m}, \mathscr{F}_{m} ; m \geqslant 1\right\}$ is a martingale. Thus, relation (3.12) follows just as above from the estimate

$$
\begin{aligned}
t_{k}^{-1} E\left|E_{k}^{*}\left(\left\langle u_{k}, T_{k-1}\right\rangle \cdot 1\left(\left|\left\langle u_{k}, T_{k-1}\right\rangle\right|>k^{-1 / 8} t_{k}\right)\right)\right| \\
\ll k^{3 / 8} t_{k}^{-4} E\left\{E_{k}^{*}\left(\left|\left\langle u_{k}, T_{k-1}\right\rangle\right|^{4}\right)\right\} \ll k^{-9 / 8} . \square
\end{aligned}
$$

LEMMA 3.8. As $k \rightarrow \infty$ we have with probability 1

$$
E_{k}^{*}\left(\left\langle u_{k}, T_{k-1}\right\rangle^{2}\right)=o\left(h_{k}\left|T_{k-1}\right|^{2}\right) \text {. }
$$

Proof. Using Lemma 3.2, Cauchy's inequality, the conditional version of Lebesgue's dominated convergence theorem, (2.5) and the orthogonality of the sequence $\left\{z_{\nu k}, \nu \geqslant 1\right\}$ with respect to $E_{k}^{*}$ we obtain

$$
\begin{aligned}
E_{k}^{*}\left(\left\langle Z_{k}, e_{i}\right\rangle\left\langle Z_{k}, e_{j}\right\rangle\right) & =\lim _{n \rightarrow \infty} E_{k}^{*}\left(\left\langle Z_{n k}, e_{i}\right\rangle\left\langle Z_{n k}, e_{j}\right\rangle\right) \\
& =\lim _{n \rightarrow \infty} \sum_{\nu=1}^{n} E_{k}^{*}\left(\left\langle z_{\nu k}, e_{i}\right\rangle\left\langle z_{\nu k}, e_{j}\right\rangle\right)
\end{aligned}
$$

To simplify notation we set $Q_{k}^{M}=\pi_{M}-\pi_{k}$ for $M>k$. We apply Lemma 3.2, Cauchy's inequality and the knowledge that $Q_{k}^{M}$ has operator norm bounded by 1 . Thus for any $H$-valued random variable $\theta$ which is measurable with respect to $\mathscr{F}_{k-1}$ and has $|\theta| \leqslant 1$, we have using (3.15) and (2.2)-(2.4), 


$$
\begin{aligned}
& E_{k}^{*}\left(\left\langle Q_{k}\left(Z_{k}\right), \theta\right\rangle^{2}\right)=\lim _{M \rightarrow \infty} E_{k}^{*}\left(\left\langle Q_{k}^{M}\left(Z_{k}\right), \theta\right\rangle^{2}\right) \\
& =\lim _{M \rightarrow \infty} E_{k}^{*}\left(\sum_{i, j=d_{k}+1}^{d_{M}}\left\langle Z_{k}, e_{i}\right\rangle\left\langle e_{i}, \theta\right\rangle\left\langle Z_{k}, e_{j}\right\rangle\left\langle e_{j}, \theta\right\rangle\right) \\
& =\lim _{M \rightarrow \infty} \sum_{i, j=d_{k}+1}^{d_{M}}\left\langle e_{i}, \theta\right\rangle\left\langle e_{j}, \theta\right\rangle E_{k}^{*}\left(\left\langle Z_{k}, e_{i}\right\rangle\left\langle Z_{k}, e_{j}\right\rangle\right) \\
& =\lim _{M \rightarrow \infty} \lim _{n \rightarrow \infty} E_{k}^{*} \sum_{\nu \leqslant n} \sum_{i, j=d_{k}+1}^{d_{M}}\left\langle e_{i}, \theta\right\rangle\left\langle e_{j}, \theta\right\rangle=E_{\nu}\left(\left\langle z_{\nu k}, e_{i}\right\rangle\left\langle z_{\nu k}, e_{j}\right\rangle\right) \\
& =\lim _{M \rightarrow \infty} \lim _{n \rightarrow \infty} E_{k}^{*} \sum_{\nu \leqslant n} \sum_{i, j=d_{k}+1}^{d_{M}}\left\langle e_{i}, \theta\right\rangle\left\langle e_{j}, \theta\right\rangle \\
& \times\left\{E_{\nu}\left\langle z_{\nu k}^{*}, e_{i}\right\rangle\left\langle z_{\nu k}^{*}, e_{j}\right\rangle-\left\langle E_{\nu} z_{\nu k}^{*}, e_{i}\right\rangle\left\langle E_{\nu} z_{\nu k}^{*}, e_{j}\right\rangle\right\} \\
& =\lim _{M \rightarrow \infty} \lim _{n \rightarrow \infty} \sum_{\nu \leqslant n}\left(E_{k}^{*}\left\langle Q_{k}^{M}(\theta), z_{\nu k}^{*}\right\rangle^{2}-E_{k}^{* 2}\left\langle Q_{k}^{M}(\theta), z_{\nu k}\right\rangle\right) \\
& \leqslant \limsup _{M \rightarrow \infty} \sum_{\nu \leqslant n} E_{k}^{*}\left\langle Q_{k}^{M}(\theta), x_{\nu k}^{*}\right\rangle^{2} \\
& =\limsup _{M \rightarrow \infty} E_{k}^{*}\left\{\left\langle Q_{k}^{M}(\theta),\left(A_{\tau(k)-1}-A_{\tau(k-1)-1}\right)\left(Q_{k}^{M}(\theta)\right)\right\rangle\right\} \\
& =\limsup _{M \rightarrow \infty} E_{k}^{*}\left\{\left\langle\theta, Q_{k}^{M}\left(A_{\tau(k)-1}-A_{\tau(k-1)-1}\right)(\theta)\right\rangle\right\} \\
& \leqslant \lim \sup E_{k}^{*}\left\|Q_{k}^{M} \cdot\left(A_{\tau(k)-1}-A_{\tau(k-1)-1}\right)\right\| \\
& \leqslant\left\|\sigma_{\tau(k-1)}\right\|+E_{k}^{*}\left\|A_{\tau(k)-1}-A_{\tau(k-1)}-\left(V_{\tau(k-1)}-V_{\tau(k-1)}\right) A\right\| \\
& +\limsup E_{k}^{*}\left\|\left(V_{\tau(k)-1}-V_{\tau(k-1)}\right) Q_{k}^{M} A\right\| \\
& =\mathrm{I}+\mathrm{II}+\mathrm{III} \text { (say). }
\end{aligned}
$$

To estimate I we note that by Lemma 2.1 and (2.2),

$$
P\left\{\left\|\sigma_{\tau(k-1)}\right\| \geqslant k^{2} f\left(t_{k}\right)\right\} \ll k^{-2} .
$$

Hence, by (3.1), (3.2) and the Borel Cantelli lemma, as $k \rightarrow \infty$,

$$
\mathrm{I}=O\left(k^{2} f\left(t_{k}\right)\right)=o\left(h_{k}\right) \text { a.s. }
$$

Observe next that

$$
\mathrm{II} \leqslant 2 f\left(V_{\tau(k)-1}\right) E_{k}^{*}\left\{\sup _{n \geqslant 1}\left\|A_{n}-V_{n} A\right\| / f\left(V_{n}\right)\right\}
$$

Further by (1.6) and the Jensen and Markov inequalities,

$$
\begin{aligned}
P\left(E _ { k } ^ { * } \left\{\sup _{n \geqslant 1} \| A_{n}-\right.\right. & \left.\left.V_{n} A \| / f\left(V_{n}\right)\right\} \geqslant 2 \log k\right) \\
& \leqslant P\left(E_{k}^{*} \exp \left\{\sup _{n \geqslant 1}\left\|A_{n}-V_{n} A\right\| / f\left(V_{n}\right)\right\} \geqslant k^{2}\right) \ll k^{-2} .
\end{aligned}
$$


Thus another application of the Borel Cantelli lemma yields that, as $k \rightarrow \infty$,

$$
\mathrm{II}=O\left(f\left(t_{k}\right) \cdot \log k\right)=o\left(h_{k}\right) \text { a.s. }
$$

For the estimate of III we apply (2.2) and obtain

$$
\mathrm{III} \leqslant \limsup _{M \rightarrow \infty} h_{k}\left\|Q_{k}^{M} A\right\|=h_{k}\left\|Q_{k} A\right\|=o\left(h_{k}\right) \quad \text { as } k \rightarrow \infty .
$$

Finally we set in (3.16)

$$
\theta= \begin{cases}T_{k-1} /\left|T_{k-1}\right| & \text { if } T_{k-1} \neq 0 \\ 0 & \text { if } T_{k-1}=0\end{cases}
$$

and obtain the lemma in view of (3.6).

Let $\rho>0$, and put

$$
W_{m}=\sum_{k \leqslant m} w_{k}
$$

and

$$
s_{m}^{2}=\sum_{k \leqslant m} \max \left(E_{k}^{*}\left(w_{k}^{2}\right), \rho^{2} h_{k} t_{k} \log \log t_{k}\right)
$$

where $w_{k}$ is defined in (3.10).

LEMMA 3.9. We have with probability 1

$$
\limsup _{m \rightarrow \infty} W_{m}\left(s_{m}^{2} \log \log s_{m}\right)^{-1 / 2} \leqslant 6 .
$$

Proof. We follow Goodman, Kuelbs and Zinn [7]. Put

$$
S_{m}=\exp \left(\lambda W_{m}-\frac{1}{2} \lambda^{2}\left(1+\frac{1}{2} \lambda c\right) s_{m}^{2}\right), \quad m \geqslant 1,
$$

where $\lambda$ and $c$ are positive constants with $\lambda c \leqslant 1$. Set

$$
m_{c}=\max \left\{k: 2 k^{-1 / 8} t_{k} \leqslant c\right\} .
$$

Since by (3.10) $w_{k} \leqslant c$ for $k \leqslant m_{c}$, Lemma 5.4.1 of Stout [11] and its proof imply that $\left\{S_{m \wedge \mu}, \mathscr{F}_{\mathrm{m}} ; m \leqslant m_{c}\right\}$ is a supermartingale for any stopping time $\mu$. Thus, by Corollary 5.4.1 of Stout [11],

$$
P\left\{\max _{m \leqslant m_{c}} S_{m \wedge \mu} \geqslant x\right\} \leqslant 1 / x, \quad x>0 .
$$

We define a sequence $\left\{\mu_{r} ; r \geqslant 1\right\}$ of stopping times:

$$
\mu_{r}=\inf \left\{l: s_{l+1}^{2} \geqslant e^{2 r}\right\} .
$$

Then $s_{\mu_{r}}^{2} \leqslant e^{2 r}$. Also, by (3.18)

$$
s_{n}^{2} \gg \rho^{2} t_{n}^{2} \log n .
$$

Hence an easy calculation gives

$$
\mu_{r} \leqslant\left(r-\frac{1}{2} \log \log r\right)^{4}+O(1) \text {. }
$$

Now put $\lambda=(\log r)^{1 / 2} e^{-r}$ and $c=1 / \lambda$. Then, by (3.1), (3.20) and (3.24), $m_{c} \geqslant \mu_{r}$ for large $r$. Therefore, by (3.19) and (3.22), 


$$
\begin{aligned}
& \limsup _{m \rightarrow \infty}\left\{W_{m}>6\left(s_{m}^{2} \log \log s_{m}\right)^{1 / 2}\right\}
\end{aligned}
$$

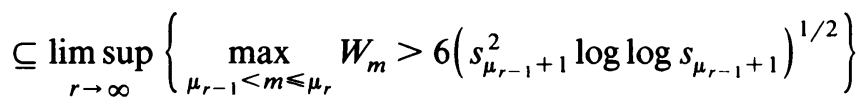

$$
\begin{aligned}
& \subseteq \limsup _{r \rightarrow \infty}\left\{\max _{m \leqslant \mu_{r}} W_{m}>5 e^{r}(\log r)^{1 / 2}\right\} \\
& \subseteq \underset{r \rightarrow \infty}{\limsup }\left\{\max _{m \leqslant m_{c}} S_{m \wedge \mu_{r}}>\exp \left(5 \log r-e^{-2 r} s_{\mu_{r}}^{2} \log r\right)\right\} .
\end{aligned}
$$

But, by (3.21)-(3.22),

$$
P\left\{\max _{m \leqslant m_{c}} S_{m \wedge \mu_{r}}>\exp \left(5 \log r-e^{-2 r} S_{\mu_{r}}^{2} \log r\right)\right\} \leqslant r^{-4} .
$$

The lemma follows now from the Borel Cantelli lemma.

We are ready to complete the proof of Proposition 2. By (3.8), (3.10), (3.17), (3.18) and Lemmas 3.6, 3.7, and 3.9 we have with probability 1

$$
\left|T_{m}\right|^{2} \ll t_{m}+s_{m}\left(\log \log s_{m}\right)^{1 / 2} .
$$

Thus by (3.23),

$$
b_{m} \stackrel{\text { def. }}{=} \max \left(\left|T_{m}\right|^{2}, \rho t_{m} \log m\right) \ll s_{m}\left(\log \log s_{m}\right)^{1 / 2} \quad \text { a.s. }
$$

But this implies by (3.10), (3.18) and Lemma 3.8

$$
\begin{aligned}
b_{m}^{2} & \ll s_{m}^{2} \log \log s_{m} \\
& \ll \rho \sum_{k \leqslant m} h_{k} \max \left(\left|T_{k-1}\right|^{2}, \rho t_{k} \log k\right) \log \log \sum_{k \leqslant m} \max \left(\left|T_{k-1}\right|^{2}, \rho t_{k} \log k\right) \\
& \ll \rho \sum_{k<m} h_{k} b_{k} \log \log \sum_{k<m} b_{k} \text { a.s. }
\end{aligned}
$$

Hence by Lemma 3.5 we have with probability 1

$$
\limsup _{m \rightarrow \infty} b_{m} /\left(t_{m} \log \log t_{m}\right) \ll \rho .
$$

Proposition 2 follows now from (3.25), (3.6) and (3.7).

LEMMA 3.10. As $m \rightarrow \infty$ we have for any Brownian motion with mean zero and covariance matrix $A$

$$
\sum_{k \leqslant m} Q_{k}\left(X\left(t_{k}\right)-X\left(t_{k-1}\right)\right)=o\left(\left(t_{m} \log \log t_{m}\right)^{1 / 2}\right) \quad \text { a.s. }
$$

This is immediate from Proposition 2 since the increments of Brownian motion satisfy the hypotheses of the Theorem.

3.3. Completion of the proof of Theorem $1(d=\infty)$. The resulting Brownian motion will be constructed as in \$2.4. First, by Remark (3.4) and Proposition 1, we obtain without loss of generality a sequence $\left\{U_{k}, k \geqslant 1\right\}$ of independent random vectors such that $U_{k}$ has Gaussian distribution $N\left(0, \pi_{k} A\right)$ and

$$
P\left\{\left|Z_{k}^{\prime}-U_{k}\right| \geqslant \alpha_{k}\right\} \leqslant \alpha_{k}
$$


(compare this with (2.18)) where by Theorem 1 of [1] and (3.1)

$$
\alpha_{k} \ll k^{-5 / 4 d_{k}}+d_{k} k^{-5 / 4} \log k+P\left\{\left|N\left(0, \pi_{k} A\right)\right| \geqslant \frac{1}{4} k^{5 / 4}\right\} \ll k^{-9 / 8} .
$$

Hence as $M \rightarrow \infty$ we have with probability 1

$$
\sum_{k \leqslant M}\left|\sum_{n \geqslant 1} h_{k}^{1 / 2} z_{n k}^{\prime}-h_{k}^{1 / 2} U_{k}\right| \ll \sum_{k \leqslant M} k^{-9 / 8} h_{k}^{1 / 2} \ll t_{M}^{1 / 2} .
$$

Thus, by the corresponding argument of $\$ 2.4$, there exists without loss of generality a Brownian motion $\{X(t) ; t \geqslant 0\}$ with mean zero and covariance matrix $A$ such that as $M \rightarrow \infty$

$$
\sum_{k \leqslant M}\left|\sum_{n \geqslant 1} h_{k}^{1 / 2} \pi_{k} z_{n k}-\pi_{k}\left(X\left(t_{k}\right)-X\left(t_{k-1}\right)\right)\right| \ll t_{M}^{1 / 2} \quad \text { a.s. }
$$

Finally, let $t \geqslant 1$ be given and define $M$ by $t_{M}<t \leqslant t_{M+1}$. Then by Lemmas 2.4, 2.5, 3.3, 3.4 and 3.10, relation (3.26), Proposition 2 and (3.1)

$$
\begin{aligned}
\mid \sum_{n \geqslant 1} x_{n} 1\left(V_{n} \leqslant\right. & t)-X(t)\left|\leqslant O(1)+h_{M+1}^{1 / 2} \sup _{n \geqslant 1}\right| Z_{n M+1} \mid \\
& +\sup _{t_{M} \leqslant t<t_{M+1}}\left|X(t)-X\left(t_{M}\right)\right|+\sum_{k \leqslant M+1} h_{k}^{1 / 2} \sum_{n \geqslant 1}\left|z_{n k}-z_{n k}^{*}\right| \\
& +\sum_{k \leqslant M}\left|\sum_{n \geqslant 1} h_{k}^{1 / 2} \pi_{k} z_{n k}-\pi_{k}\left(X\left(t_{k}\right)-X\left(t_{k-1}\right)\right)\right| \\
& +\left|\sum_{k \leqslant M} h_{k}^{1 / 2} Q_{k}\left(Z_{k}\right)\right|+\left|\sum_{k \leqslant M} Q_{k}\left(X\left(t_{k}\right)-X\left(t_{k-1}\right)\right)\right| \\
= & o\left(\left(t_{M} \log \log t_{M}\right)^{1 / 2}\right)=o\left((t \log \log t)^{1 / 2}\right) \text { a.s. }
\end{aligned}
$$

\section{REFERENCES}

1. István Berkes and Walter Philipp, Approximation theorems for independent and weakly dependent random vectors, Ann. Probability 7 (1979), 29-54.

2. B. M. Brown, Martingale central limit theorems, Ann. Math. Statist. 42 (1971), 59-66.

3. D. L. Burkholder, Distribution function inequalities for martingales, Ann. Probability 1 (1973), 19-42.

4. Herold Dehling and Walter Philipp, Almost sure invariance principles for weakly dependent vector-valued random variables, preprint, 1980.

5. X. Fernique, Integrabilité des vectours Gaussiens, C. R. Acad. Sci. Paris 270 (1970), 1698-1699.

6. Peter Gänssler and Winfried Stute, Wahrscheinlichkeitstheorie, Springer-Verlag, Berlin and New York, 1977.

7. V. Goodman, J. Kuelbs and J. Zinn, Some results on the LIL in Banach space with applications to the weighted empirical process, Ann. Probability 9 (1981), 713-752.

8. Naresh C. Jain, Kumar Jogdeo and William F. Stout, Upper and lower functions for martingales and mixing processes, Ann. Probability 3 (1975), 119-145.

9. N. C. Jain and S. J. Taylor, Local asymptotics for Brownian motion, Ann. Probability 1 (1973), 527-549.

10. J. Kuelbs and R. LePage, The law of the iterated logarithm for Brownian motion in a Banach space, Trans. Amer. Math. Soc. 185 (1973), 253-264.

11. J. Kuelbs and Walter Philipp, Almost sure invariance principles for partial sums of mixing B-valued random variables, Ann. Probability 8 (1980), 1003-1036.

12. S. Orey and W. E. Pruitt, Sample functions of the $N$-parameter Wiener process, Ann. Probability 1 (1973), 138-163. 
13. Walter Philipp and William F. Stout, Almost sure invariance principles for partial sums of weakly dependent random variables, Mem. Amer. Math. Soc. No. 161 (1975).

14. William F. Stout, Almost sure convergence, Academic Press, New York, 1974.

15. V. Strassen, An almost sure invariance principle for the law of the iterated logarithm, Z. Wahrscheinlichkeitstheorie Verw. Gebiete 3 (1964), 211-226.

16. Almost sure behavior of sums of independent random variables and martingales, Proc. Fifth Berkeley Sympos. Math. Stat. Prob., Vol. 2, 1965, pp. 315-342.

Department of Mathematics, Washington University, St. Louis, Missouri 63130

DePaRTMEnt of Mathematics, University of Illinois, Urbana, Illinois 61801 